\title{
La evolución de la calidad hacia la calidad total
}

\section{The evolution of quality towards total quality}

1 Ronny Pedro Carriel Sevillano

Universidad de Guayaquil, Guayaquil, Ecuador ronny.carriels@ug.edu.ec

2 Albert Joao Nieto Pacheco

Universidad de Guayaquil, Guayaquil, Ecuador albert.nietop@ug.edu.ec
(iD) https://orcid.org/0000-0003-2484-1325

https://orcid.org/0000-0001-5296-5574

Artículo de Investigación Científica y Tecnológica

Enviado: $12 / 12 / 2021$

Revisado: $27 / 12 / 2021$

Aceptado: 28/01/2022

Publicado:05/02/2022

DOI: https://doi.org/10.33262/ap.v4i1.1.166

Carriel Sevillano, R. P., \& Nieto Pacheco, A. J. (2022). La evolución de la calidad hacia
Cítese:
la
calidad
total
AlfaPublicaciones,
4(1.1),
383-397.

https://doi.org/10.33262/ap.v4i1.1.166

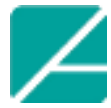

Ciencia

Digital
ALFA PUBLICACIONES, es una Revista Multidisciplinar, Trimestral, que se publicará en soporte electrónico tiene como misión contribuir a la formación de profesionales competentes con visión humanística y crítica que sean capaces de exponer sus resultados investigativos y científicos en la misma medida que se promueva mediante su intervención cambios positivos en la sociedad. https://alfapublicaciones.com

La revista es editada por la Editorial Ciencia Digital (Editorial de prestigio registrada en la Cámara Ecuatoriana de Libro con No de Afiliación 663) www.celibro.org.ec

Esta revista está protegida bajo una licencia Creative Commons Attribution Non Commercial No Derivatives

4.0 International. Copia de la licencia: http://creativecommons.org/licenses/by-nc-nd/4.0/ 
Palabras

claves:

calidad total, gestión, mejora continua.

\section{Keywords:}

total quality, management, continuous improvement.

\section{Resumen}

La presente investigación se enfoca en realizar una revisión minuciosa de los conceptos que envuelven a la calidad, así como de la evolución de estos conceptos y lo que se ha logrado llevar a cabo gracias a la adopción de sistemas de gestión de calidad en diversas áreas de las empresas que han decidido aplicar. Los conceptos van desde las primeras eras del hombre hasta el presente siglo XXI en el cual se evidencia un avance significativo en cuanto a teorías se refiere. De igual manera se enfoca la investigación en revisar las diversas teorías que hasta la fecha se han podido obtener de los grandes pioneros y expertos en el área de la calidad, haciendo particular énfasis en la teoría de la Calidad Total, en la cual convergen diversos puntos descritos por otras teorías, pero esta vez enfocadas en toda la organización, bajo la premisa de una mejora continua y verificación y estandarización de procesos mediante técnicas y herramientas estadísticas y de control.

\section{Abstract}

The present research focuses on conducting a thorough review of the concepts that surround quality, as well as the evolution of these concepts and what has been achieved thanks to the adoption of quality management systems in various areas of the companies that have decided to apply. The concepts range from the first eras of man to the present XXI century in which a significant advance in terms of theories is evidenced. In the same way, the research is focused on reviewing the various theories that to date have been obtained from the great pioneers and experts in the area of quality, with particular emphasis on the theory of Total Quality, in which various points converge described by other theories but this time focused on the entire organization, under the premise of continuous improvement and verification and standardization of processes through statistical and control techniques and tools.

\section{Introducción}

El ser humano desde sus inicios en el planeta tierra ha presentado necesidades básicas, comida, sustento, abrigo y morada, esto sin duda movió al hombre a la búsqueda de mejoras en sus condiciones de vida, estas mejoras estaban ligadas para ese entonces a la funcionalidad que pudiesen brindar, solo se buscaba la supervivencia del ser humano. 
Con el pasar de los siglos el hombre y sus descubrimientos permitieron conocer y desarrollar más y mejores técnicas que desencadenaron un auge en la manera de cómo elaborar productos, que bajo la creciente explosión demográfica que sufría el planeta para esas épocas resultaba necesario mejorar los métodos artesanales de producción, conocido como la época de la industrialización, en esta etapa la economía del planeta planteaba nuevos retos y los consumidores ya no se limitaban a solo cubrir una necesidad, sino que progresivamente se fueron añadiendo características y exigencias sobre los productos y en respuesta a esto las empresas se vieron en la necesidad y obligación de adaptar sus procesos y productos a la satisfacción del cliente.

La economía mundial se ha vuelto muy competitiva ya que los avances en materia de tecnología han provocado una carrera por cumplir los estándares exigidos por los mercados actuales, esto aunado al fenómeno de la globalización en donde todos los mercados compiten con productos más innovadores y con altos estándares de calidad se hace necesario que las empresas tomen nuevas metodologías para cubrir las altas demandas de los clientes. Esto ha provocado que las empresas del siglo XXI sean dinámicas y capaces de competir a nivel global, bajo un esquema normalizado aceptado por la gran mayoría de los países del mundo, esta normalización la proporciona la calidad y sus diferentes vertientes o teorías.

En esta investigación se pretende dar un bosquejo de la evolución de los conceptos de la calidad, sus principales características y las teorías que han surgido para mejorar los procesos industriales y de servicios, todo esto bajo un enfoque sistémico de calidad, tomando como referente la calidad total, ya que es la teoría que se adapta de una mejor manera a la visión de un mundo globalizado y con una creciente demanda de procesos eficientes y eficaces que proporcionen seguridad y confianza en los productos y servicios.

\section{Metodología}

La investigación es de tipo documental, se realizó una revisión bibliográfica entre documentos de orden científico, revistas de investigaciones, tesis de grado, documentos de organismos internacionales emitidos bajo los más estrictos estándares científicos académicos, investigaciones científicas que proporcionan la información necesaria para realizar la documentación del tema. La investigación documental, como herramienta ayuda en la construcción del conocimiento, amplia los constructos hipotéticos de los estudiantes y como enriquece su vocabulario para interpretar su realidad desde su disciplina, constituye elemento motivador para la realización de procesos investigativos (Gómez et al., 2016).

Con este método se pretende establecer las comparativas respectivas en los temas que se tratan en la investigación. 


\section{Resultados y Discusión}

Esta ocurre por decisiones de la alta gerencia con el fin de lograr un nivel adecuado de La calidad es un concepto ampliamente manejado desde los tiempos antiguos, cuando los hombres comenzaron a confeccionar sus propias ropas e indumentaria para cubrir sus cuerpos, a elaborar sus propios utensilios para la caza y la pesca, entre otras labores que implicaban la satisfacción de ciertas necesidades, aunque básicas pero que debían mantener un estándar de funcionalidad y utilidad que pudiese resolver los problemas de comida y vestido para ese entonces, ya allí se comienza a denotar una satisfacción o cumplimiento de estándares, que para ese caso específico puede mencionarse que cumplía estándares de funcionalidad, el concepto de calidad ha ido evolucionando desde la prehistoria hasta la actualidad, pero alcanza su mayor relevancia en la actividad empresarial y en la última mitad del siglo anterior Becerra et al. (2019), con la llegada de la era de la máquina de vapor muchos fueron los avances que en materia de tecnología se avanzó, tanto que se mecanizaron todos o casi todos los procesos industriales que anteriormente se realizaban a baja escala y de manera muy artesanal, de igual manera se procedió a estandarizar las formas y maneras de producir ciertos productos, por ejemplo se habla de la estandarización de las piezas, la cual se sobrevino por la producción en masa que permitió la industrialización, la cual no es más que la necesidad de producir piezas de algún artefacto que cumpliese con los estándares requeridos por los ingenieros que diseñaron dicho artefacto, las piezas que componen cierta máquina o aparato deben ser iguales y precisas en sus dimensiones ya que el ensamblaje debe ser prolijo y cumplir con el funcionamiento del mismo, esto marco la diferencia entre los procesos artesanales y los industrializados, precisamente por la necesidad de establecer los parámetros de producción bajo los cuales debía operar un proceso.

Progresivamente, el concepto de calidad ha ido evolucionando desde las primeras eras de la tierra, la Organización Internacional de Normalización (2015), ISO por sus siglas en ingles en su norma ISO9000:2015 define calidad como grado en el que un conjunto de características inherentes de un objeto cumple con los requisitos dichos requisitos se encuentran definidos por la misma norma como necesidad o expectativa establecida, generalmente implícita u obligatoria, de igual manera se definen los requisitos de calidad como el requisito relativo o inherente a la calidad. Por lo cual se compone el concepto de calidad como un cumplimiento de un producto o servicio a los requisitos previamente establecidos por el cliente o por la organización, según Amaya et al. (2020) menciona que:

Un cliente queda satisfecho si se le ofrece todo lo que él esperaba encontrar y más. Así, la calidad, es ante todo la satisfacción del cliente, la cual está ligada a las expectativas que éste tiene sobre el producto o servicio; y tales 
expectativas son generadas de acuerdo con necesidades, antecedentes, precio, publicidad, tecnología, imagen de la empresa, entre otros aspectos.

De igual manera, Huacon \& Arzube (2019) la describe como, "la suma de los productos más útiles solicitados por los clientes" otro concepto de uno de los pioneros en el tema de la calidad lo describe por los mismos autores, pero esta vez citan a Juran (1993) y la define como algo apropiado para usarse. Dichas definiciones dan su enfoque hacia la satisfacción del cliente, en el cual se plantea como única premisa a cumplir los estándares fijados o esperados por el cliente, esto sin embargo da pie a las evoluciones y avances que ha tenido el concepto de calidad desde que los especialistas pioneros en esta área comenzaros a dar sus primeros conceptos y definiciones de la misma. La calidad está asociada con la intensión de que un producto final pueda ser usado por los clientes y este genere una sensación de satisfacción al momento de ser usado, de igual manera cuando se habla de calidad explícitamente se habla de excelencia, bien sea la fabricación de un producto o la prestación de un servicio.

Se puede decir que la calidad tiene los siguientes significados según Benzaqende las Casas (2018) mostrado en la figura 1.

\section{Figura 1}

\section{Decálogo de la Calidad}

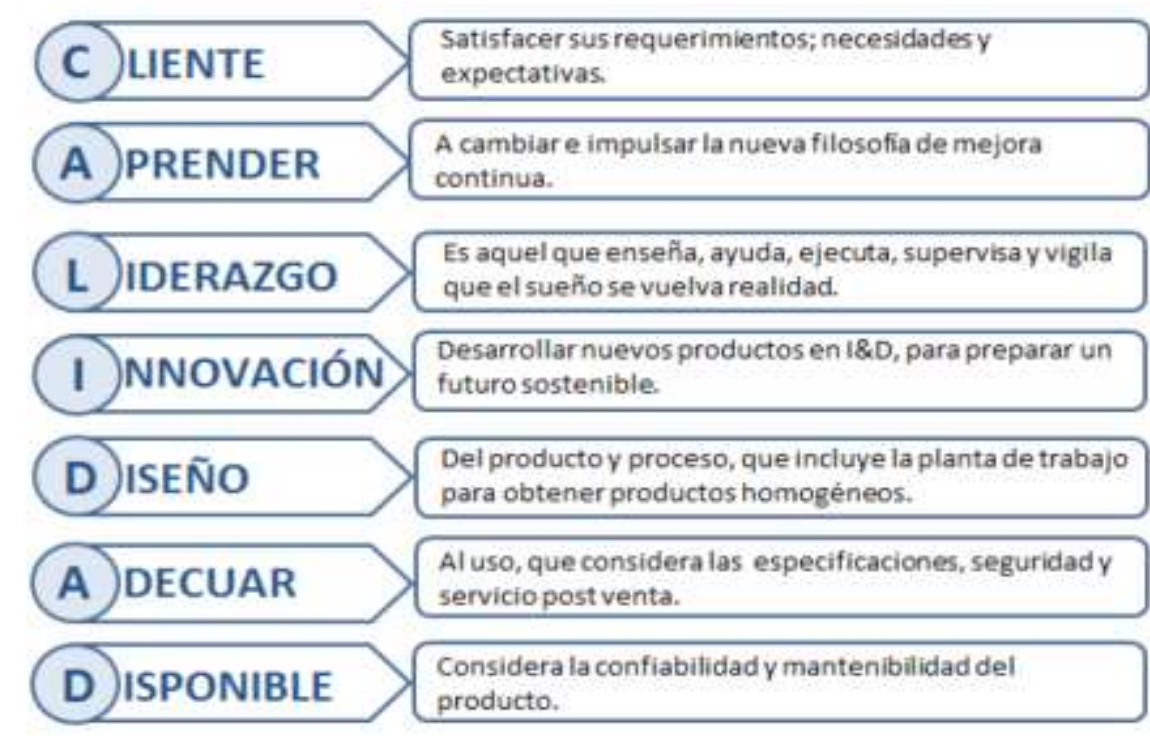

Fuente: Benzaqende las Casas (2018)

Tal como se puede visualizar en la figura anterior, la calidad lleva implícitos ciertas características que complementan el solo hecho se satisfacer las demandas de ciertos clientes, implica el aprendizaje continuo para la aplicación de las mejoras en todo proceso que se decida normalizar o aplicar criterios de calidad, lleva inmersa una de las cualidades más importantes que debe poseer toda persona que pretende impulsar las políticas de 
calidad en una organización, y es que el liderazgo juega un papel fundamental para poder realizar todos los cambios necesarios que implica la implantación de los preceptos de calidad, igualmente los conceptos de diseño, innovación y adecuación de los procesos y productos para que se adapten a las exigencias y estándares preestablecidos.

La calidad es una parte muy importante en todo proceso productivo y de servicios, aunque inicialmente solo se aplicaba a la producción en las empresas de manufactura, este concepto fue trasladado a empresas que prestaban algún tipo de servicios, abarcando así casi cualquier tipo de actividad productiva, no es sino dar un vistazo a los grandes resultados que ha proporcionado las teorías e innovaciones que se han logrado por la sistematización de los procesos bajo un enfoque sistémico y controlado bajo premisas de excelencia y calidad, aportando estándares cada vez más altos y adicionando mejoras sustanciales en los procesos.

Según Prieto (2017) la calidad parte de tres premisas fundamentales:

- En cualquier empresa, toda situación precedente o actual es mejorable.

- En la situación actual de competencia creciente y globalizada, el juez único del nivel de calidad alcanzado por cualquier empresa son los clientes y los consumidores de sus productos.

- Cualquier empresa que ignore en su estrategia y en su gestión el juicio, las necesidades y las expectativas de sus clientes y consumidores, fracasará.

En esta contextualización, se puede entender que la calidad es un proceso que conlleva a evolucionar sobre el camino andado previamente, lo que da a la calidad un plus para las empresas que decidan asumir el proceso de implantación de calidad en sus procesos, pero debe ser asumida desde la alta gerencia de la organización y están conscientes de capacitar al capital humano en esta materia y contar con un equipo líder experto que pueda llevar por el camino de la calidad a los demás empleados, este equipo debe contar con amplia experiencia en el tema y debe ser versátil para poder adecuar los procesos y de esta manera instaurar en los empleados el sentido de mejora continua propio del personal que trabaja con calidad.

La calidad en su concepto más simple, lleva a quien quiera aplicarla hacia un punto de antes y después, es sin duda alguna una de las estrategias que mejor ha llevado a cabo los progresos de adecuación de procesos y manipulación de variables en una organización, ha logrados establecer los controles necesarios que han llevado al éxito a múltiples empresas a nivel mundial, tal es el caso del caso del Japón. Según American Society for Quality (ASQ, 2022):

Al principio, Japón tenía una reputación generalizada de exportaciones de mala calidad y sus productos fueron rechazados por los mercados 
internacionales. Esto llevó a las organizaciones japonesas a explorar nuevas formas de pensar sobre la calidad. Las estrategias de Japón representaron el nuevo enfoque de calidad total. En lugar de depender únicamente de la inspección de productos, los fabricantes japoneses se centraron en mejorar todos los procesos organizativos a través de las personas que los utilizaban. Como resultado, Japón pudo producir exportaciones de mayor calidad a precios más bajos, beneficiando a los consumidores de todo el mundo.

La filosofía que aplico Japón trajo como consecuencia una revolución de la percepción de los productos a nivel mundial, tanto así cambio su imagen la aplicación de los principios de la calidad, que hoy día hablar de calidad es sinónimo de Japón y sus múltiples ejemplos de superación y vuelcos totales a la calidad de los productos, esto sin duda fue un ejemplo a seguir para países como los Estados Unidos de Norteamérica, quienes pensaban y afirmaban que elevar los estándares de calidad de los productos elevaría los costos de los productos y no fue así, fue una teoría que revocaron los japoneses y la respuesta del país norteamericano fue adoptar y aplicar las teorías de calidad impulsadas y ampliamente aplicadas por el Japón.

Luego de toda esta avanzada en materia de calidad y donde los conceptos más básicos evolucionaron para dar paso a nuevas metodologías de trabajo bajo el enfoque de calidad surgen diversas teorías que maduraron en el transcurrir del tiempo y desde la experiencia, se puede ver en la tabla 1 las diferentes teorías que fueron planteadas por los padres de la calidad a lo largo de los años.

\section{Tabla 1}

Filosofías y teorías de la calidad

\begin{tabular}{|c|c|c|}
\hline Autor & Filosofía/Teoría Aplicada & Conceptos Principales \\
\hline $\begin{array}{l}\text { Edwards Deming } \\
(1900-1993) \\
\text { Estadístico } \\
\text { Estadounidense }\end{array}$ & $\begin{array}{l}\text { Control estadístico de la calidad. } \\
\text { "CALIDAD TOTAL" } \\
\text { Ciclo PHVA (planificar, hacer, } \\
\text { verificar y actuar) o PDCA }\end{array}$ & $\begin{array}{l}\text { Control estadístico de procesos } \\
\text { Filosofía de Administración para la } \\
\text { Calidad. } \\
\text { Ciclo PDCA. } \\
\text { Mejorar para ser competitivos }\end{array}$ \\
\hline $\begin{array}{l}\text { Joseph M. Juran } \\
(1954) \\
\text { Ingeniero, Abogado } \\
\text { y asesor rumano. }\end{array}$ & $\begin{array}{l}\text { "TRILOGIA DE JURAN" } \\
\text { • Planificación de la } \\
\\
\text { Calidad. } \\
\text { • Control de la Calidad. } \\
\text { • Mejora de la Calidad }\end{array}$ & $\begin{array}{l}\text { Definir mercado y su necesidad. } \\
\text { Definir características del producto y } \\
\text { servicio a lograr. } \\
\text { Desarrollo de Procesos Productivos. } \\
\text { Cumplir expectativas/conformidad. } \\
\text { Comparar producto vs Objeticos de } \\
\text { Calidad, actuar sobre las diferencias, } \\
\text { establecer metas para la mejora continua, } \\
\text { Realizar proyectos para solucionar } \\
\text { problemas, planificar el alcance de las } \\
\text { metas, registrar y comunicar resultados. }\end{array}$ \\
\hline
\end{tabular}




\section{Tabla 1}

Filosofías y teorías de la calidad (continuación)

\begin{tabular}{|c|c|c|c|}
\hline Autor & Filosofía/Teoría Aplicada & & Conceptos Principales \\
\hline $\begin{array}{l}\text { Kaoru Ishikawa } \\
\text { (1915-1989) } \\
\text { Ingeniero, } \\
\text { catedrático, } \\
\text { consultor japonés. } \\
\text { Presidente Japonés } \\
\text { ante ISO } \\
\text { Premio Deming y } \\
\text { de Normalización } \\
\text { Industrial. } \\
\text { Medalla Sehwhart }\end{array}$ & $\begin{array}{l}\text { Normalización Industrial para } \\
\text { fortalecer la productividad. } \\
\text { Siete herramientas técnicas } \\
\text { (Estadística de análisis de } \\
\text { problema). } \\
\text { Cuadro de Pareto. } \\
\text { Diagrama causa efecto } \\
\text { (Diagrama de Ishikawa) } \\
\text { Estratificación } \\
\text { Hoja de verificación } \\
\text { Histogramas } \\
\text { Diagrama de dispersión } \\
\text { Gráficas y cuadro de control. }\end{array}$ & - & $\begin{array}{l}\text { Primero la calidad luego la } \\
\text { utilidad. } \\
\text { El cliente es lo más importante } \\
\text { (orientación hacia el cliente). } \\
\text { Prevenir no corregir. } \\
\text { Trabajo en Equipo. } \\
\text { Compromiso de la Alta } \\
\text { dirección. } \\
\text { Resultados a largo plazo (no } \\
\text { hay caminos cortos para } \\
\text { alcanzar la calidad) } \\
\text { Medir resultados. } \\
\text { Dar reconocimientos } \\
\text { Proceso de Mejora continua. }\end{array}$ \\
\hline
\end{tabular}

\begin{tabular}{|c|c|c|}
\hline $\begin{array}{l}\text { Phillip B. Crosby } \\
\text { (1980’s) }\end{array}$ & $\begin{array}{l}\text { "Cero defectos" } \\
\text { Proceso de mejoramiento de la } \\
\text { calidad en } 14 \text { pasos. } \\
\text { Cultura preventiva. } \\
\text { Indica que los errores se } \\
\text { producen por falta de } \\
\text { conocimiento, de atención o de } \\
\text { ambos. Sugiere un cambio de } \\
\text { mentalidad para lograr el } \\
\text { estándar de cero defectos }\end{array}$ & $\begin{array}{l}\text { Cuatro principios de la Calidad, según } \\
\text { Crosby. } \\
\text { Calidad se define como cumplir con los } \\
\text { requisitos. } \\
\text { El sistema de calidad es la prevención El } \\
\text { estándar de la realización es Cero Defectos } \\
\text { La medida de la calidad es el precio del } \\
\text { cumplimiento }\end{array}$ \\
\hline $\begin{array}{l}\text { TQM- Total Quality } \\
\text { Management } \\
\text { (1960`s) }\end{array}$ & $\begin{array}{l}\text { Teoría de la Calidad Total. } \\
\text { No se considera una herramienta } \\
\text { de medición estadística, ni un } \\
\text { proceso de control de calidad, } \\
\text { sino una cultura de calidad y } \\
\text { cambio organizacional. }\end{array}$ & $\begin{array}{l}\text { Se centra en: } \\
\text { Mejoramiento continuo } \\
\text { Medición de la calidad } \\
\text { Cambio de cultura organizacional } \\
\text { Liderazgo } \\
\text { En la década de los 90, unido con CQI } \\
\text { (Continuos QualityImprovement) } \\
\text { promovió el programa TQM/CQI, con } \\
\text { profundo impacto en el sistema de salud } \\
\text { canadiense. (Price, } 1994 \text { ) }\end{array}$ \\
\hline $\begin{array}{l}\text { Kaizen. Ventaja } \\
\text { Competitiva } \\
\text { japonesa (1960’s) }\end{array}$ & $\begin{array}{l}\text { Teoría de la Calidad Total de los } \\
\text { japoneses Kaizen sugiere que la } \\
\text { variación de la calidad tiene } \\
\text { impacto directo en los costos y } \\
\text { en la gente. }\end{array}$ & $\begin{array}{l}\text { Indica que la cultura de la calidad se centra } \\
\text { en la calidad de las personas, luego de los } \\
\text { productos. Implica el ciclo E.H.R.A. } \\
\text { (estandarizar, hacer, revisar y actuar) Su } \\
\text { enfoque es transversal, y es una adaptación } \\
\text { del ciclo de Deming. }\end{array}$ \\
\hline
\end{tabular}


La anterior información solo denota el auge e importancia que ha tomado la calidad y sus diferentes enfoques según los autores citados, los cuales han sido los pioneros en el tema de calidad a nivel mundial y en diferentes épocas.

Dentro de las teorías más interesantes de calidad se encentra la de calidad total, la cual envuelve una serie de preceptos que se enfocan en normalizar, como su nombre lo indica la totalidad de los diferentes procesos que conforman una organización.

La calidad total y la cultura del mejoramiento continuo son metas organizacionales que permiten brindar una mayor satisfacción al usuario de un servicio o sistema, en el marco de sus políticas organizacionales. La implantación de un Modelo de Calidad tiene un papel transformador otorgando un valor agregado al servicio, con su impacto en la eficiencia organizacional, mejoramiento continuo, control o reingeniería de procesos y optimización de recursos, aumento del desempeño y productividad (Chacón \& Rugel, 2018).

Para Comisión, Medici (2020) proponen tres enfoques para resumir la evolución del concepto gestión de la calidad:

- El enfoque técnico; Se centra en la eficiencia de los productos y procesos de la empresa, recoge el concepto de empresa orientada al cumplimiento de las especificaciones

- El enfoque humano; El soporte de este enfoque es tratar de lograr que los trabajadores sean el eje del proceso de gestión y mejora de la calidad a través de su participación en la solución de problemas.

- El enfoque estratégico; Se basa en la evaluación no sólo de los procesos y actividades internas de la organización, sino también en los efectos que dichos procesos y actividades generan sobre los grupos de interés de la empresa: accionistas, empleados, clientes, proveedores y comunidad en general.

En la tabla 2 se resumen los enfoques:

Tabla 2

Evolución de la gestión de la calidad

\begin{tabular}{ll}
\hline Enfoques & Desarrollo \\
\hline $\begin{array}{l}\text { Enfoque estratégico } \\
\text { (Desde años 80) }\end{array}$ & $\bullet$ Gestión de Calidad Total \\
& $\bullet$ Esfuerzo de búsqueda de excelencia en toda la \\
& organización y el sistema de valor. \\
& $\bullet$ Compaginar eficiencia interna con eficacia. \\
\hline Enfoque humano & $\bullet$ Cambio cultural de la \\
(Años 70-90) & organización \\
& $\bullet$ Innovaciones del diseño organizativo \\
& $\bullet$ Liderazgo y estilo de dirección
\end{tabular}




\section{Tabla 2}

Evolución de la gestión de la calidad (continuación)

\begin{tabular}{ll}
\hline Enfoques & Desarrollo \\
\hline Enfoque humano & $\bullet$ Actividades de resolución de problemas \\
(Años 70-90) & $\bullet$ Participación directa de los trabajadores \\
& $\bullet$ Círculos de calidad y equipos de mejora \\
& $\bullet$ Formación de los empleados. \\
\hline & ETAPAS \\
Enfoque técnico & $\bullet$ Orientación a la prevención \\
(Hasta años 90) & $\bullet$ Orientación al sistema \\
& $\bullet$ Orientación al proceso \\
& $\bullet$ Orientación al producto \\
\hline
\end{tabular}

Fuente: Medici (2020)

Los enfoques por los que ha transitado la calidad total solo denota la maduración de los conceptos que la componen, adaptándose a las características y necesidades de los tiempos en que fueron concebidas dichas teorías, con el pasar de los años y el transcurrir de las experiencias en diversas áreas en donde se ha aplicado la filosofía de calidad total se han evidenciado aportes significativos a las empresas en las que se ha aplicado, seguidamente se listan una serie de aportes que han hecho de la calidad total un referente obligado a la hora de habla de innovación, estos aportes son mencionados por (Sánchez et al., 2020).

- La Gestión de Calidad Total (CGT) como modelo de gestión multidimensional, tiene prácticas con un enfoque mecanicista que se vinculan con el desempeño de calidad, y prácticas con un enfoque orgánico relacionadas con el desempeño de la innovación.

- Aunque GCT es un medio más eficaz para lograr resultados de calidad que de innovación, contribuye a esta última a veces explícitamente y, en la mayoría de los casos, de forma implícita, por lo que puede ser utilizada en conjunto con GT/ I+D para mejorar el desempeño de la innovación.

- Si se quiere alcanzar un alto nivel de desempeño de la innovación se debe tener la capacidad para gestionar por completo los requisitos de calidad.

- Los principios de GCT son eficaces para el desarrollo de capacidades en las áreas de I+D y pueden facilitar la estandarización de los nuevos productos, procesos o servicios.

- La GCT puede proporcionar un entorno adecuado para superar posibles barreras y acrecentar la capacidad de innovación de las organizaciones por su potencial para desarrollar cambios en la cultura, si se aplica en sentido amplio y no se centra solamente en los aspectos técnicos. 
- La GCT debe ser complementada con otros recursos existentes en la organización para apoyar de manera más eficaz la consecución de un alto nivel de desempeño en toda la gama de prácticas de innovación.

- La GCT permite la explotación de las capacidades de la empresa mediante el desarrollo de innovaciones incrementales y promueve el desarrollo de competencias necesarias para la innovación radical.

- La GCT, si se alinea con otras estrategias, podría proveer una base sobre la cual las organizaciones pueden construir sus competencias y capacidades tecnológicas para lograr una ventaja competitiva multidimensional que incluya la innovación, así como la capacidad de adopción y absorción de esta dentro de la empresa.

- Se presupone una relación compleja entre GCT e innovación tecnológica (considerada como la innovación basada en la aplicación industrial de conocimientos científicos y tecnológicos) que requiere seguir siendo estudiada para definir con mayor especificidad el impacto de cada una de las diferentes prácticas de GCT en la innovación y su alineación a la GT/I+D en diferentes contextos.

Como todo método o proceso, la calidad total tiene ciertas características que lo definen y diferencian de las demás propuestas o enfoques de teorías de la calidad, para Rodríguez (2018) destacan las siguientes Características:

- Un control de los errores que se van cometiendo para edificar un sistema que los evite antes de que sucedan.

- Consiste en el apoyo total de la dirección de la empresa hacia una dinámica de mejora en la calidad y productividad

- Se trata de medir la calidad con instrumentos o métodos adecuados y conseguir que cada una de las personas de la empresa se sienta comprometida personalmente en una nueva forma de trabajar hacia la fiabilidad

- Asegurar en cada fase del proceso productivo que las acciones llevadas a cabo y las características incorporadas en el producto van a contribuir a un resultado final que esté conforme con las necesidades del cliente

- Establecer un lenguaje y unas comunicaciones tales entre personas y empresas que permita transmitir los conceptos sin que existan malentendidos

- Optimización continua del proceso de forma que se vaya reduciendo la variabilidad de los parámetros que intervienen

- Tolerancia cada vez inferior en cuanto a las variaciones del nivel de calidad final de la imagen de los productos.

- Un seguimiento estadístico de los resultados obtenidos, a ser posible por Departamento, para valorar el éxito del progreso

- Cumplir con los plazos de entrega convenidos con el cliente 
- Disponer de una atmósfera de cordialidad y trato satisfactorio con proveedores y clientes.

Para Prieto (2017) la calidad total supone ciertas necesidades de cumplir con algunos requisitos para su implementación en cualquier organización y estaría enfocada en lo siguiente:

- La calidad "sale" del área de producción y se va extendiendo progresivamente a todas las funciones y áreas de la empresa.

- No es suficiente que un proceso sea bueno, todos tienen que ser eficientes, eficaces y asegurar la calidad objetivo.

- Todo el personal de la empresa (directivos, ejecutivos, mandos intermedios y trabajadores) deben participar activamente en la consecución del nivel de calidad objetivo.

- Dinamismo continuo y mejora continua, en base al siguiente ciclo.

Asimismo, se muestra este proceso en la figura 2

\section{Figura 2}

\section{Requisitos para ser implementados en cualquier organización}

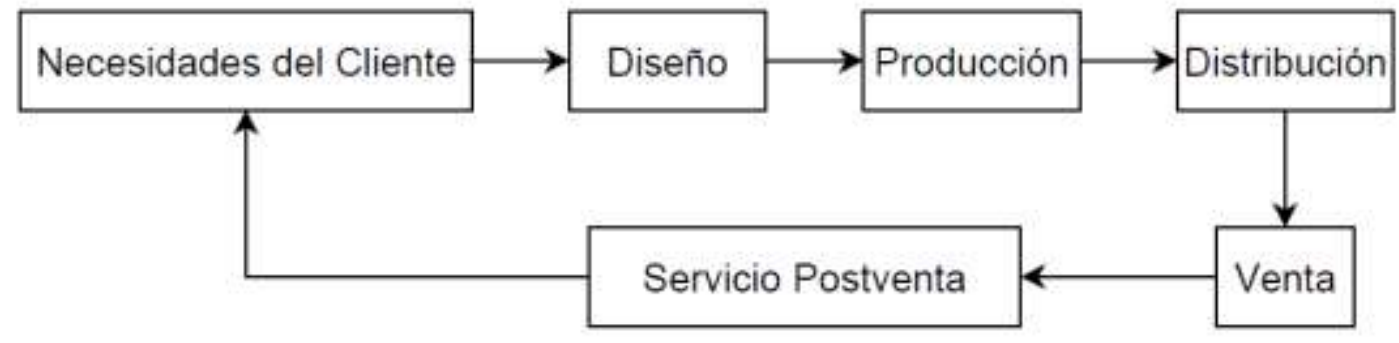

Fuente: Prieto (2017)

De tal manera que la calidad total no es más que un conjunto de técnicas enfocadas a la satisfacción no solo del cliente, sino de los clientes internos, que son quienes llevan a cabo la realización de los productos o servicios que se prestan, esto hace que todos los niveles que conforman la organización sean responsables directos de hacer que sus procesos sean de excelencia, intentando ser más productivos día a día por medio del enfoque de mejora continua, el cual permita resolver las inconformidades de un proceso o producto, esto sin duda ha logrado que las empresas u organizaciones hoy día posean procesos totalmente estandarizados en función del cumplimiento de requisitos previamente establecidos por la organización o los clientes. Las expectativas de calidad son altas hoy en día y la competitividad está ligada a la excelencia en los servicios y productos que se ofrezcan, no en vano más empresas se suman a la filosofía de la calidad como premisa fundamental en sus misiones y visiones como organizaciones. 


\section{Conclusiones}

- La calidad no es un concepto nuevo, desde los tiempos antiguos fue necesario la verificación de la utilidad de muchos utensilios e indumentaria para satisfacer las necesidades básicas de los primeros hombres del planeta, al pasar de los siglos y con los descubrimientos de los hombres se fue perfilando un método simple de verificación, que hoy día se conoce como inspección y forma parte de los componentes de la calidad.

- Luego, en la era de la industrialización fue posible la producción en masa, lo cual condujo a la estandarización de piezas o componentes, que también acarreó la estandarización de los procesos, esto sin duda marco un antes y un después en lo que hoy se conoce como calidad, ya que la estandarización de procesos en una de las características fundamentales de la calidad en todo sentido y aspecto, los grandes pensadores de la época como Deming, Juran, Ishikawa entre otros fueron hilando teorías de calidad que aportaban un peldaño más a los conceptos que se manejaban hasta esas épocas sobre la calidad, estos enfoques se fueron dando a medida que las filosofías de mejora continua surtían efecto en las empresas u organizaciones que decidieron creer en la calidad e implantaron poco a poco en sus sistemas de producción los principios fundamentales de la calidad. En este contexto, la filosofía japonesa tuvo un gran impacto en revocar creencias de costos elevados derivados de la calidad, resultaron en procesos industriales óptimos, con menos perdidas y con productos de excelente calidad.

- Más adelante se evoluciona hacia el concepto de Calidad Total, el cual enfoca de una manera sistémica los principios de la calidad, pero aplicándolo a todos los niveles de las organizaciones, esto con la finalidad de lograr productos y servicios de oprima calidad y procesos administrativos estandarizados en la que se logre la producción de una manera eficiente y eficaz.

\section{Referencias Bibliográficas}

Amaya, P., Félix, E., Rojas, S., \& Diaz, L. (2020). Gestión de la Calidad, un estudio desde sus principios. Revista Venezolana de Gerencia, 632-647.

American Society for Quality, ASQ. (2022). Learn About Quality.

Becerra, F., Andrade, A., \& Diaz, L. (2019). Sistema de gestión de la calidad para el proceso de investigación: Universidad de Otavalo, Ecuador. Revista Electrónica “Actualidades Investigativas en Educación”, 1-32.

Benzaqende las Casas, J. (2018). La ISO 9001 y la Administración de la Calidad Total en las Empresas Peruanas. Universidad y Empresas 20(35), 281-312. 
Chacón, J., \& Rugel, S. (2018). Articulo de Revisión. Teorías, modelos y Sistemas de Gestión de la Calidad. Revista Espacios, 14.

Gómez, D., Carranza, Y., \& Ramos, C. (2016). Revisión Documental, Una Herramienta para el Mejoramiento de las Competencias de Lectura y Escritura en Estudiantes Universitarios. UNACH Revista Chakinan, 45-56.

Huacon, G., \& Arzube, I. (2019). La Evolución de la productividad y calidad en las empresas de bienes y servicios. Revista Observatorio de la Economía Latinoamericana.

Medici, L. (2020). ISO 9000: Evolución hacia la calidad total. Publicaciones en Ciencia y tecnología, 3-13.

Organización internacional de normalización (ISO). (2015). ISO 9000:2015(es); Sistemas de gestión de la calidad - Fundamentos y vocabulario.

Prieto, J. (2017). Guía Para Master en Sistemas Integrados de Gestión de Calidad Medio Ambiente y Prevención de Riesgos laborales. Madrid: Universidad Nacional de Educación a Distancia.

Rodríguez, J. (2018). Calidad Total.

Sánchez, L., Yépez, N., \& Pedroza, A. (2020). La relación entre gestión de la calidad total $(G C T)$ y gestión de la tecnología $/ I+D(G T / I+D)$ en empresas de manufactura en México. Contaduría y Administración

\section{【Liencia}


El artículo que se publica es de exclusiva responsabilidad de los autores y no necesariamente reflejan el pensamiento de la Revista Alfa Publicaciones.

\section{Ciencia}

El artículo queda en propiedad de la revista y, por tanto, su publicación parcial y/o total en otro medio tiene que ser autorizado por el director de la Revista Alfa Publicaciones.
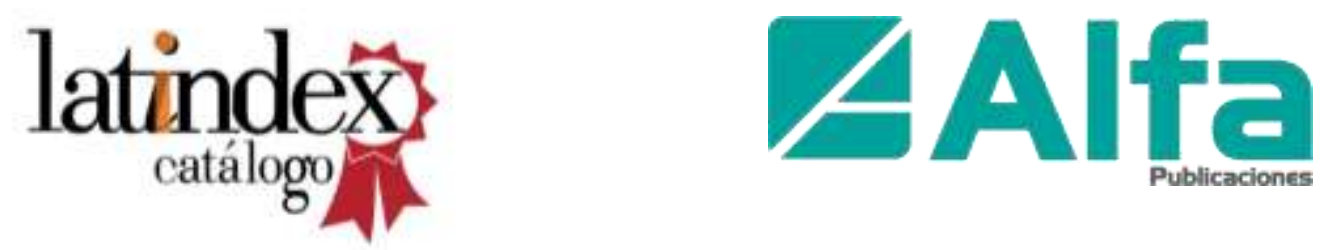

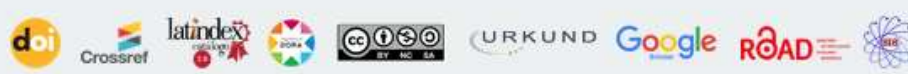
DLatinREV

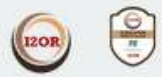

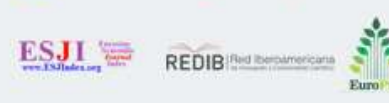

$\underbrace{}_{\text {wizdom.ai }} \mathrm{O}_{\text {OpenAIRE }}^{+}$

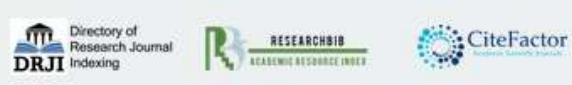

